\title{
Evaluación positiva de medicamentos: Diciembre 2013, Enero y Febrero 2014
}

\author{
Pérez Morán MJ. ${ }^{1}$, Prats Olivan P. ${ }^{1}$, Tabacov A. ${ }^{2}$, García Luque A. ${ }^{3}$ \\ Sanid. mil. 2014; 70 (2): 98-102; ISSN: 1887-8571
}

RESUMEN

Se reseñan los medicamentos ya evaluados por la Agencia Española de Medicamentos y Productos Sanitarios, considerados de mayor interés para el profesional sanitario en el ámbito hospitalario. Se trata de opiniones técnicas positivas que son previas a la autorización y puesta en el mercado del medicamento.

Positive assessment of drugs: December 2013 January, February 2014

SUMMARY: The drugs assessed by the Spanish Agency for Medicines and Health Products made public in, and considered of interest in hospital healthcare professional, are reviewed. These are positive technical reports prior to the authorization and placing on the market of the product.

\section{ALBIGLUTIDA $\left(\text { EPERZAN }{ }^{\circledR}\right)^{1,2}$}

El principio activo es albiglutida, un agonista del receptor GLP-1 (péptido similar al glucagón 1), generado mediante la fusión de un análogo de GLP-1 a la albúmina, lo que da lugar a una vida media mucho más prolongada. Al igual que el GLP-1 originario, la albiglutida mejora la secreción de insulina glucosadependiente y reduce la liberación de glucagón.

En los ensayos clínicos, albiglutida ha mostrado un efecto clínico relevante sobre el control glucémico en pacientes con diabetes tipo 2, cuando se emplea junto con otros medicamentos hipoglucemiantes como la insulina, o en monoterapia, cuando no se puede emplear la metformina. La albiglutida tiene un efecto neutro sobre el peso corporal.

Indicado en el tratamiento de la diabetes mellitus tipo 2 en adultos para mejorar el control glucémico. En monoterapia: cuando la dieta y el ejercicio por sí solos no logran un control glucémico adecuado en pacientes en quienes el uso de metformina se considera inadecuado por presentar intolerancia o contraindicaciones. Como adición a la terapia de combinación: En combinación con otros medicamentos reductores de la glucosa, como la insulina basal, cuando estos junto con dieta y ejercicio, no logren un control glucémico adecuado (ver secciones 4.4 y 5.1 de la ficha técnica sobre información disponible de las diferentes combinaciones).

Las reacciones adversas observadas con más frecuencia durante el desarrollo clínico fueron: náuseas, diarrea y reacciones en el lugar de la inyección.

1a Cap. Farmacéutico.

1a Cte. Farmacéutico.

${ }^{2 b}$ Médico Interno Residente (R3) de Farmacología clínica.

${ }_{3 \mathrm{~b}}$ Cte. Médico.

a Servicio de Farmacia hospitalaria.

b Servicio de Farmacología clínica.

Hospital Central de la Defensa Gómez Ulla. Madrid. España.

Dirección para correspondencia: Servicio de Farmacia Hospitalaria. Hospital Central de la Defensa Gómez Ulla. 28047 Madrid. España. mpermor@et.mde.es

Recibido: 4 de marzo de 2014

Aceptado: 14 de marzo de 2014

\section{BEDAQUILINA $\left(\text { SIRTURO }{ }^{\circledR}\right)^{3,4}$}

El principio activo es bedaquilina, una diarilquinolina que actúa inhibiendo específicamente la ATP sintetasa de la micobacteria, una enzima esencial para la producción de energía de Mycobacterium tuberculosis. Esta inhibición produce efectos bactericidas tanto para bacilos tuberculosos en fase replicativa como en fase no replicativa.

La bedaquilina ha mostrado una actividad clínicamente relevante contra la tuberculosis como parte de un tratamiento de combinación. Según los datos clínicos, el tratamiento con bedaquilina se mostró eficaz en términos de conversión del cultivo de esputo en pacientes con tuberculosis multirresistente, definidos como resistentes al menos a los dos principales medicamentos tuberculostáticos (isoniazida y rifampicina) que recibieron bedaquilina hasta 24 semanas en combinación con un régimen de base compuesto por otros medicamentos para el tratamiento de la MDR-TB.

Está indicada en la tuberculosis pulmonar multirresistente (MDR-TB) como parte de un tratamiento de combinación en pacientes adultos cuando otra combinación eficaz no puede administrarse por razones de resistencia o tolerabilidad. Se deben considerar las recomendaciones oficiales sobre el uso adecuado de agentes antibacterianos.

El Comité Europeo de Evaluación de Medicamentos (CHMP) ha recomendado de manera condicional la autorización de comercialización de Sirturo, al satisfacer una necesidad médica no cubierta, en la medida en que, el beneficio para la salud pública de su inmediata disponibilidad es superior al riesgo inherente de que todavía se requieran datos adicionales. El titular de la autorización de comercialización proporcionará más datos clínicos posteriormente.

Un estudio de un solo brazo actualmente en marcha, que también incluye pacientes con tuberculosis extremadamente resistente a los medicamentos (XDR-TB), ha proporcionado datos adicionales de eficacia.

Los estudios parecen indicar que actualmente, la bedaquilina no tiene resistencia cruzada a los medicamentos antituberculosos disponibles. 


\section{Evaluación positiva de medicamentos: Diciembre 2013, Enero y Febrero 2014}

Como medidas postautorización, se solicitan estudios adicionales para definir mejor el uso óptimo de este medicamento, tanto en lo que respecta al número y tipo de fármacos que se necesitan en combinación, como a la duración óptima del tratamiento.

La reacción adversa observada con más frecuencia durante el desarrollo clínico fue el aumento de las enzimas hepáticas. Como en el caso de otros fármacos antituberculosos, las enzimas hepáticas se deben controlar durante el tratamiento de la tuberculosis.

También se observó prolongación del intervalo QTc durante el tratamiento con bedaquilina. Debe hacerse seguimiento de la prolongación del QTc y evaluar el beneficio-riesgo cuando se combinen bedaquilina y otros fármacos antimicobacterianos que también prolonguen significativamente el intervalo QTc.

Se recomienda su prescripción por médicos con experiencia en el tratamiento de la tuberculosis pulmonar multirresistente. Bedaquilina fue designado como medicamento huérfano el 26 de agosto de 2005.

\section{CABOZANTINIB (COMETRIQ $\left.{ }^{\circledR}\right)^{3,5}$}

El principio activo es cabozantinib, un agente antineoplásico inhibidor de la proteína quinasa. Actúa inhibiendo múltiples receptores de tirosina quinasas implicados en el crecimiento tumoral y angiogénesis, remodelación ósea patológica y metástasis. Al inhibir los receptores de tirosina quinasas, cabozantinib podría enlentecer o detener el crecimiento del cáncer tiroideo medular.

En los ensayos clínicos, cabozantinib ha mostrado que incrementa la supervivencia libre de progresión en comparación con placebo en pacientes con carcinoma tiroideo medular progresivo, no resecable localmente avanzado o metastásico.

Tratamiento de pacientes adultos con carcinoma tiroideo medular progresivo, no resecable, localmente avanzado o metastásico. En pacientes en los que no se conozca el estatus del protooncogen RET (reorganizado durante la transfección) o sea negativo, se debe tener en cuenta que el beneficio puede ser menor antes de tomar una decisión individual de tratamiento.

El Comité Europeo de Evaluación de Medicamentos (CHMP) ha recomendado de manera condicional su autorización de comercialización, al satisfacer una necesidad médica no cubierta, en la medida en que, el beneficio para la salud pública de su inmediata disponibilidad es superior al riesgo inherente de que todavía se requieran datos adicionales. El titular de la autorización de comercialización proporcionará más datos clínicos posteriormente.

Las reacciones adversas observadas con más frecuencia durante el desarrollo clínico fueron: diarrea, síndrome de eritrodisestesia palmo-plantar, disminución de peso, anorexia, náuseas, vómitos, fatiga, disgeusia, cambios en el color del pelo, hipertensión, estomatitis, estreñimiento, mucositis, astenia y disfonía. En cuanto a las alteraciones analíticas, las más comunes fue-ron aumento de AST, ALT, ALP, linfopenia, hipocalcemia, hipofosfatemia, hiperbilirrubinemia, neutropenia, trombocitopenia e hipoalbuminemia.

Se recomienda su prescripción por médicos con experiencia en la administración de tratamientos oncológicos. Cabozantinib fue designado como medicamento huérfano el 26 de febrero de 2009.

\section{CANAGLIFLOZINA / METFORMINA (VOKANAMET $\left.{ }^{\circledR}\right)^{6,7}$}

Es una combinación de dos principios activos que actúan como agentes hipoglucemiantes, con mecanismos de acción diferentes, canagliflozina y metformina. Canagliflozina añadido a metformina ha mostrado que confiere una mejoría adicional clínicamente relevante en el control glucémico, lo que justifica la combinación de estas sustancias.

Canagliflozina es un inhibidor reversible del cotransportador sodio-glucosa tipo 2 (SGLT-2), que reduce la reabsorción de glucosa a nivel renal, aumentando su eliminación en orina y reduciendo de esta forma la glucemia. La eliminación aumentada de glucosa renal produce una diuresis osmótica, y también resulta en una pérdida de calorías, contribuyendo a la reducción de peso corporal. La metformina actúa mediante la supresión de la producción de glucosa en el hígado. Indicado en adultos de 18 años de edad o mayores con diabetes mellitus tipo 2 como adyuvante a la dieta y el ejercicio para mejorar el control glucémico:

- En pacientes no controlados adecuadamente con la dosis máxima tolerada de metformina en monoterapia.

- En pacientes con dosis máxima tolerada de metformina en combinación con otros medi-camentos hipoglucemiantes incluyendo insulina, cuando éstos no consiguen un control glu-cémico adecuado.

- En pacientes que ya se están tratando con la combinación de canagliflozina y metformina en comprimidos separados.

Las reacciones adversas más comunes de la metformina son síntomas gastrointestinales tales como náuseas, vómitos, diarrea, dolor abdominal y pérdida de apetito. Las reacciones adversas más comunes de canagliflozina son hipoglucemia (cuando se usa en combinación con insulina o sulfonilurea), candidiasis vulvovaginal, infección del tracto urinario, y poliuria o polaquiuria.

Se recomienda su prescripción por médicos con experiencia en el tratamiento de la diabetes mellitus tipo

\section{ELOSULFASA ALFA (VIMIZIM® $)^{6,8}$}

La elosulfasa alfa es una $\mathrm{N}$-acetilgalactosamina-6 sulfatasa humana recombinante. Elosulfasa alfa suplementaría el déficit de N-acetilgalactosamina-6 sulfatasa. Tras la administración intravenosa la enzima es absorbida por los lisosomas y se produce un aumento del catabolismo de los glicosaminoglicanos.

Está indicada en el tratamiento de mucopolisacaridosis tipo IV A (Síndrome de Morquio) en pacientes de todas las edades.

En los ensayos clínicos se ha observado que mejora el test de la marcha de los 6 minutos, y que mejora otros síntomas de la enfermedad de Morquio, como las funciones respiratorias o antropométricas.

Las reacciones adversas observadas con más frecuencia durante el desarrollo clínico fueron reacciones de infusión, incluyendo anafilaxis, hipersensibilidad y vómitos.

El tratamiento debe ser supervisado por un médico con experiencia en el tratamiento de pacientes con mucopolisacaridosis tipo IV A u otras enfermedades metabólicas hereditarias. La 
administración debe hacerse por un profesional sanitario con experiencia en el manejo de urgencias médicas. Vimizin fue designado como medicamento huérfano el 24 de julio de 2009.

\section{FLORBETABEN (18F) (NEURACEQ® $)^{3,9}$}

Este medicamento es únicamente para uso diagnóstico.

Neuraceq es un radiofármaco indicado para la obtención de imágenes mediante tomografía por emisión de positrones (PET) de la densidad de placa neurítica de $\beta$-amiloide en el cerebro de pacientes adultos con deterioro cognitivo que están siendo evaluados por enfermedad de Alzheimer (EA) y otras causas de deterioro cognitivo. Neuraceq debe usarse en combinación con la evaluación clínica.

El principio activo es florbetaben $(18 \mathrm{~F})$, un radiofármaco indicado para la obtención de imágenes mediante tomografía por emisión de positrones (PET) de la densidad de placa neurítica de $\beta$-amiloide.

En los ensayos clínicos ha mostrado su capacidad para estimar la deposición de beta-amiloide y por tanto contribuir con información adicional al proceso clínico de diagnóstico de la EA.

Las reacciones adversas observadas con más frecuencia durante el desarrollo clínico fueron reacciones en el sitio de inyección: irritación y dolor.

Neuraceq debe prescribirse por médicos con experiencia en el manejo clínico de enfermedades neurodegenerativas. Las imágenes de Neuraceq deben interpretarlas lectores entrenados en la interpretación de imágenes PET con florbetaben $(18 \mathrm{~F})$.

\section{FOLITROPINA ALFA $\left(\text { BEMFOL } A^{\circledR}\right)^{1,10}$}

Bemfola es un biosimilar de Gonal-f (folitropina alfa).

La Folitropina alfa es una hormona foliculoestimulante humana recombinante, esencial para el crecimiento normal y maduración de los gametos femeninos y para la inducción de la producción esteroide gonadal normal. La deficiencia endógena en la producción de FSH es una causa conocida de infertilidad y la administración de gonadotropinas de manera exógena se utiliza para tratar esta enfermedad.

Indicaciones aprobadas:

En mujeres adultas:

- Anovulación (incluyendo el síndrome de ovario poliquístico) en mujeres que no han respondido al tratamiento con citrato de clomifeno.

- Estimulación del desarrollo folicular múltiple en mujeres sometidas a superovulación para realizar técnicas de reproducción asistida (TRA), tales como la fertilización in vitro (FIV), transferencia intratubárica de gametos y transferencia intratubárica de cigotos.

- Folitropina alfa, asociado a un preparado de hormona luteinizante (LH), se recomienda para la estimulación del desarrollo folicular en mujeres con deficiencia severa de LH y FSH. En los ensayos clínicos, estas pacientes se definieron por un nivel sérico de LH endógena de $<1,2 \mathrm{UI} / 1$.
En varones adultos:

- Para estimular la espermatogénesis en varones con hipogonadismo hipogonadotropo congénito o adquirido, administrado de forma concomitante con Gonadotropina Coriónica humana (hCG).

Las reacciones adversas observadas con más frecuencia durante el desarrollo clínico fueron: cefalea, quistes ováricos y reacciones locales en el sitio de inyección. Se ha observado frecuentemente el síndrome de hiperestimulación ovárica (SHO) leve o moderado y se debe considerar como un riesgo intrínseco del procedimiento de estimulación. Muy raramente podría ocurrir tromboembolismo, normalmente asociado a síndrome de hiperestimulación ovárica ( $\mathrm{SHO}$ ) grave.

Se recomienda su prescripción por médicos con experiencia en el tratamiento de los problemas de infertilidad.

\section{LURASINODA $\left(\text { LATUDA }{ }^{\circledR}\right)^{1,11}$}

Lurasidona es un antipsicótico que bloquea los efectos de dopamina y monoamina. Se une principalmente a receptores dopaminergicos D2 y a los receptores serotoninérgicos 5-HT2A y 5-HT7. Esto afecta a la actividad del cerebro y reduce los síntomas de la esquizofrenia (síntomas psicóticos).

En los ensayos clínicos, ha mostrado que mejora los síntomas psicóticos en comparación con placebo.

Ha sido aprobado para el tratamiento de la esquizofrenia en pacientes adultos.

Las reacciones adversas observadas con más frecuencia durante el desarrollo clínico fueron: acatisia y somnolencia.

\section{PROPRANOLOL (HEMANGIOL $\left.{ }^{\circledR}\right)^{6,12}$}

Propranolol es conocido betabloqueante. Los potenciales mecanismos de acción para el tratamiento del hemangioma infantil proliferativo descritos en la literatura incluyen efecto hemodinámico local, efecto antiangiogénico, efecto inductor de la apoptosis sobre las células del endotelio capilar o una reducción de las rutas de transducción de señales de VEGF y bFGF.

Esta indicado en el tratamiento del hemangioma infantil proliferativo que requiera terapia sistémica:

- Hemangioma con riesgo vital o funcional.

- Hemangioma ulcerado con dolor o falta de respuesta a medidas simples de cuidado de la herida.

- Hemangioma con riesgo de cicatrices o desfiguración.

El tratamiento se debe iniciar en niños de 5 semanas a 5 meses de edad.

En los ensayos se ha observado la resolución del hemangioma a tratar de una manera completa o casi completa, evaluado de forma independiente, centralizada y ciega sobre fotografías a la semana 24 de tratamiento.

Las reacciones adversas observadas con más frecuencia durante el desarrollo clínico fueron: alteraciones del sueño, agrava- 
miento de infecciones del tracto respiratorio, como bronquitis y bronquiolitis asociadas con tos y fiebre, diarrea y vómitos.

Se recomienda su prescripción por médicos con experiencia en el diagnóstico, tratamiento y manejo del hemangioma infantil.

\section{RIOCIGUAT $(A D E M P A S \circledR)^{1,13}$}

Riociguat es un estimulador de la guanilato ciclasa soluble (GCs), una enzima del sistema cardiopulmonar y receptor para el oxido nítrico (NO). Cuando el NO se une a la GCs, la enzima cataliza la síntesis de la molécula de señalización guanosina monofosfato cíclica (GMPc). El GMPc intracelular juega un papel importante en los procesos de regulación que influyen en el tono vascular, la proliferación, la fibrosis y la inflamación.

Riociguat sensibiliza la GCs al NO endógeno mediante la estabilización de la unión NO-GCs y estimula directamente al GCs independientemente del NO.

Las Indicaciones aprobadas: son Hipertensión pulmonar tromboembólica crónica (HTPTC).

También está indicado para el tratamiento de pacientes adultos clasificados con clase funcional II a III de la OMS para mejorar la capacidad de ejercicio en, HTPTC inoperable, HTPTC persistente o recurrente después del tratamiento quirúrgico, Hipertensión arterial pulmonar (HAP).

Como monoterapia o en combinación con antagonistas de los receptores de la endotelina, está indicado para el tratamiento de pacientes adultos con hipertensión arterial pulmonar (HAP) clasificados como clase funcional II a III de la OMS, para mejorar la capacidad de ejercicio.

Se ha demostrado la eficacia de este medicamento en HAP idiopática o hereditaria y en HAP asociada a enfermedad del tejido conectivo.

Las reacciones adversas observadas con más frecuencia durante el desarrollo clínico fueron: cefalea, hipotensión, alteraciones gastrointestinales, mareos y edema periférico. Otras reacciones adversas de interés fueron hemoptisis, hemorragia pulmonar e insuficiencia renal considerando las diferencias que se observaron frente al grupo de placebo.

Se recomienda su prescripción por médicos con experiencia en el tratamiento de la HTPTC y HAP.

\section{TARTRATO DE BRIMONIDINA (MIRVASO $\left.{ }^{\circledR}\right)^{3,14}$}

El tartrato de brimonidina es un agonista relativamente selectivo de los receptores alfa-2 adrenérgicos, con una potente actividad vasoconstrictora y vasoestabilizadora. El eritema de la rosácea está ligado a la vasodilatación permanente de pequeños vasos. La reducción del eritema facial se consigue gracias a la vasoconstricción mediada por la estimulación de los receptores alfa-2 adrenérgicos postsinápticos del músculo liso.

Esta indicado en el tratamiento sintomático del eritema facial de la rosácea en adultos.

En los ensayos clínicos el tartrato de brimonidina ha mostrado superioridad sobre un vehículo tipo gel con respecto al inicio rápido de un efecto clínico significativo (evaluación del eritema por el clínico y por el paciente) a los 30 minutos tras la primera aplicación en el día 1, y alcanzar un efecto clínico significativo a las 3, 6, 9 y 12 horas del día 29 tras la administración.

Las reacciones adversas locales observadas con más frecuencia durante el desarrollo clínico fueron: eritema, prurito, rubefacción y sensación de quemazón.

\section{TRAVOPROST $(I Z B A \AA)^{3,15}$}

Travoprost es un análogo de prostaglandina F2 $\alpha$, agonista selectivo de los receptores de prostaglandinas, que reduce la presión intraocular mediante el aumento del drenaje.

Está indicado en la disminución de la presión intraocular elevada en pacientes adultos con hipertensión ocular o glaucoma de ángulo abierto.

En los ensayos clínicos, travoprost ha mostrado que reduce la hipertensión intraocular con una eficacia similar a la alcanzada con la concentración de $40 \mu \mathrm{g} / \mathrm{ml}$, actualmente en el mercado.

Las reacciones adversas observadas con más frecuencia durante el desarrollo clínico fueron a nivel ocular: hiperemia, irritación, dolor, prurito en los párpados y sensación de cuerpo extraño.

\section{UMECLIDINIO (INCRUSE®) ${ }^{6,16}$}

Umeclidinio es un antagonista del receptor muscarínico de acción prolongada (LAMA), que ejerce su actividad broncodilatadora inhibiendo competitivamente la unión de la acetilcolina con los receptores muscarínicos de acetilcolina en el músculo liso de las vías respiratorias.

Los beneficios de umeclidinio se basan en su capacidad de mejorar el FEV1 (variable de función pulmonar) y mejorar los síntomas de EPOC medido por los cambios en la puntuación en una escala de disnea (TID) y en la escala de calidad de vida específica de EPOC (SGRQ).

Está indicado como tratamiento broncodilatador de mantenimiento para aliviar los síntomas en pacientes adultos con enfermedad pulmonar obstructiva crónica (EPOC).

Las reacciones adversas observadas con más frecuencia durante el desarrollo clínico fueron nasofaringitis e infecciones del tracto respiratorio superior. Un problema potencial de seguridad de umeclidinio es el aumento en la incidencia de acontecimientos cardiovasculares y cerebrovasculares que pueden ocurrir con el uso a largo plazo. Este riesgo se evaluará en profundidad en estudios posautorización diseñados para tal objetivo.

\section{UMECLIDINIO/VILANTEROL ( ANOROI LAVENTAIR $\left.{ }^{\circledR}\right)^{6,17}$}

Es un medicamento formado por una combinación a dosis fijas del principio activo umeclidinio, un antagonista del receptor muscarínico de acción prolongada (también conocido como anticolinérgico), y el principio activo vilanterol, un agonista selectivo del receptor beta 2- adrenérgico de acción prolongada.

Los antagonistas de los receptores muscarínicos de acción prolongada ejercen su actividad broncodilatadora inhibien- 


\section{MJ. Pérez Morán, et al.}

do competitivamente la unión de la acetilcolina con los receptores muscarínicos de acetilcolina en el músculo liso de las vías respiratorias. Los agonistas de los receptores beta 2-adrenérgicos estimulan la adenilato ciclasa intracelular que convierte el ATP en AMP cíclico. Este aumento de los niveles de AMP cíclico provoca la relajación del músculo liso bronquial y la inhibición de la liberación de mediadores de hipersensibilidad in-mediata de las células, especialmente de los mastocitos. Los beneficios en el tratamiento de EPOC se basan en su capacidad de mejorar la función pulmonar medida por el FEV1 (variable de función pulmonar) y los síntomas de disnea, medidos por el cambio en la puntuación en la escala TID a la semana 24 en un estudio controlado frente a placebo.

Indicado como tratamiento broncodilatador de mantenimiento para aliviar los síntomas en pacientes adultos con enfermedad pulmonar obstructiva crónica (EPOC).

Las reacciones adversas observadas con más frecuencia durante el desarrollo clínico fueron nasofaringitis y dolor de cabeza. Un problema potencial de seguridad es el aumento en la incidencia de acontecimientos cardiovasculares y cerebro vasculares que pueden ocurrir con el uso a largo plazo. Este riesgo se evaluará en profundidad en estudios post autorización diseñados para tal objetivo.

\section{BIBLIOGRAFIA}

1. Agencia Española de Medicamentos y Productos Sanitarios. Informe mensual sobre medicamentos de Uso Humano y Productos Sanitario. Enero 2014. Disponible en: http://www.aemps.gob.es/informa/boletinMensual/2014/enero/docs/boletin-mensual_enero-2014.pdf

2. Committee for Medicinal Products for Human Use (CHMP) EMA/ CHMP/20616/2014 23 January 2014. Disponible en: http://www.ema.europa. eu/docs/en_GB/document_library/Summary_of_opinion_-_Initial_authorisation/human/002735/WC500159960.pdf

3. Agencia Española de Medicamentos y Productos Sanitarios. Informe mensual sobre medicamentos de Uso Humano y Productos Sanitario. Diciembre 2013. Disponible en: http://www.aemps.gob.es/informa/boletinMensual/2013/diciembre/docs/boletin-mensual_diciembre-2013.pdf

4. Committee for Medical Products for Human Use (CHMP) EMA 771324/201 19 December 2013 Disponible en: http://www.ema.europa.eu/docs/en_GB/ document_library/Summary_of_opinion_-_Initial_authorisation/human/002614/WC500158728.pdf

5. Committee for Medicinal Products for Human Use (CHMP) EMA/ CHMP/539526/2013 19 december 2013. Disponible en: http://www.ema.
europa.eu/docs/en_GB/document_library/Summary_of_opinion_-_Initial_ authorisation/human/002640/WC500158674.pdf

6. Agencia Española de Medicamentos y Productos Sanitarios. Informe mensual sobre medicamentos de Uso Humano y Productos Sanitario Febrero 2014. Disponible en: http://www.aemps.gob.es/ informa/boletinMensual/2014/febrero/docs/boletin-mensual_febrero-2014.pdf

7. Committee for Medicinal Products for Human Use (CHMP) EMA/ CHMP/90545/2014 20 february 2014 http://www.ema.europa.eu/docs/ en_GB/document_library/Summary_of_opinion_-_Initial_authorisation/human/002656/WC500161964.pdf

8. Committee for medical products for human use (CHMP) EMA/ CHMP/92845/2014 20 february 2014. Disponible en: http://www.ema.europa.eu/docs/en_GB/document_library/Summary_of_opinion_-_Initial_ authorisation/human/002779/WC500161935.pdf

9. Committee for medical products for human use (CHMP). EMA/ CHMP/772521/2013 19 december 2013. Disponible en: http://www.ema. europa.eu/docs/en_GB/document_library/Summary_of_opinion_-_Initial_ authorisation/human/002553/WC500158677.pdf

10. Committee for medical products for human use (CHMP). EMA/ CHMP/41421/2014 23 january 2014 Disponible en: http://www.ema.europa. eu/docs/en_GB/document_library/Summary_of_opinion_-_Initial_authorisation/human/002615/WC500159959.pdf

11. Committee for medical products for human use (CHMP). EMA/ CHMP/26583/2014 23 january 2014. Disponible en: http://www.ema.europa. eu/docs/en_GB/document_library/Summary_of_opinion_-_Initial_authorisation/human/002713/WC500160099.pdf

12. Committee for medical products for human use (CHMP). EMA/CHMP 95120/2014 20 febrero 2014 . Disponible en: http://www.ema.europa.eu/docs/ en_GB/document_library/Summary_of_opinion_-_Initial_authorisation/human/002621/WC500161926.pdf

13. Committee for Medicinal Products for Human Use (CHMP) EMA CHMP807324/2013 23 January 2013. Disponible en: http://www.ema.europa. eu/docs/en_GB/document_library/Summary_of_opinion_-_Initial_authorisation/human/002737/WC500160094.pdf

14. Committee for Medicinal Products for Human Use (CHMP) EMA/ CHMP0684313/2013 19 december 2013. Disponible en: http://www.ema. europa.eu/docs/en_GB/document_library/Summary_of_opinion_-_Initial_ authorisation/human/002642/WC500158676.pdf

15. Committee for Medicinal Products for Human Use (CHMP) EMA/CHMP 592024/2013 19 December 2013. Disponible en: http://www.ema.europa.eu/ docs/en_GB/document_library/Summary_of_opinion_-_Initial_authorisation/human/002738/WC500158675.pdf

16. Committee for medical products for human use (CHMP) EMA/CHMP 68182/2014 20 February 2014. Disponible en: http://www.ema.europa.eu/ docs/en_GB/document_library/Summary_of_opinion_-_Initial_authorisation/human/002809/WC500161966.pdf

17. Committee for medical products for human use (CHMP) EMA/CHMP 55884/2014 20 February 2014. Disponible en: http://www.ema.europa.eu/ docs/en_GB/document_library/Summary_of_opinion_-_Initial_authorisation/human/002751/WC500161956.pdf 